\title{
Are the New ACR/EULAR Criteria the Ultimate Answer for Polymyalgia Rheumatica Classification?
}

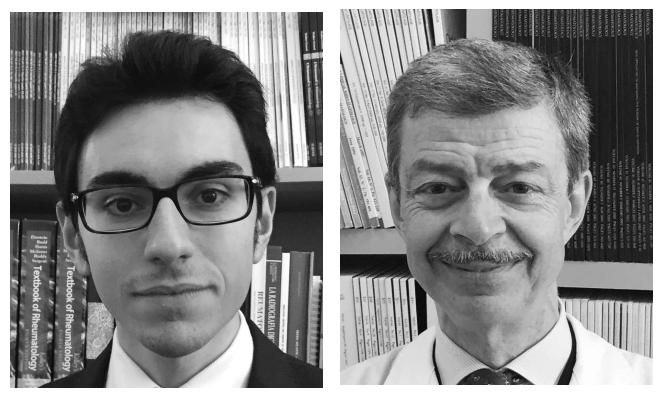

Polymyalgia rheumatica (PMR) is a diagnostic limbo. If a patient is evaluated by an experienced clinician who records the usual set of signs and symptoms along with the pertinent increased inflammatory markers, the diagnosis is usually correct. However, in several instances, PMR may evolve or transform into elderly onset rheumatoid arthritis (EORA), a switch that cannot be easily predicted and is usually recognized only during followup ${ }^{1}$. When less experienced clinicians are involved, overdiagnosis and underdiagnosis of PMR are relatively frequent because several conditions may mimic the disease, and a gold standard for diagnosis confirmation is lacking.

The availability of the recent American College of Rheumatology/European League Against Rheumatism (ACR/EULAR) criteria is expected to improve PMR classification ${ }^{2}$. Clinicians may also be enticed to apply these criteria in the individual patient although, as widely stated ${ }^{3}$, criteria should be used for classification only. The multifaceted essence of PMR may account for the wide range of its clinical presentations, making it difficult to identify a 1 -size-fits-all set of criteria.

In this issue of The Journal, Ozen, et $a l^{4}$ describe a multicenter study dealing with the comparison of different sets of PMR classification criteria, including the ACR/EULAR criteria. The main findings of this study, which confirmed the overall good performance of the criteria, are that they are not optimal in differentiating PMR from seronegative polyarthritis and that, if a cutoff for laboratory inflammation is included, performance can increase. By comparing the existing sets of criteria, several studies from the literature obtained different results (Table $1^{2,4,5,6,7,8,9}$ ). In Ozen's study, the new ACR/EULAR criteria showed a moderate-to-good discriminating capacity between PMR and non-PMR. However, the best performance was obtained by the Chuang, et al criteria ${ }^{10}$, also in the case of differentiation between PMR and EORA. In accordance with previous findings, the Bird $^{9}$, et al criteria showed the best sensitivity $(94 \%)$, while those of Jones and Hazleman ${ }^{11}$ and of Chuang, et al, showed the highest specificity. The unexpected finding of Ozen's study is that the ACR/EULAR criteria showed more sensitivity than specificity, contrary to what was observed in the original article ${ }^{2}$ and in Macchioni, et al ${ }^{6}$ (Table 1). In addition, specificity of the ACR/EULAR criteria increased when they were used to differentiate PMR from RA (but not from its seronegative subset), again in disagreement with the original study, which showed better performance when the criteria were used to discriminate between PMR and noninflammatory shoulder diseases. This observation is surprising, because EORA is unanimously considered the most challenging differential diagnosis.

Is PMR the same disease for all clinicians? It is ironic that even if the same ingredients are used, the resulting cocktail is so unpredictable. In fact, there is an overlap of $80 \%$ in the different items of the 6 most frequently used criteria. In particular, age over 50 years is considered in $4 / 6$, elevated erythrocyte sedimentation rate (ESR) or C-reactive protein (CRP) in 6/6, morning stiffness in 4/6, bilateral shoulder pain and stiffness in $6 / 6$, and exclusion of other conditions, especially peripheral arthritis in 5/6. However, the definition of the single item is somewhat different. The nature of the patients, control group population, and clinicians who apply the criteria may further explain some of the discrepancies. In the ACR/EULAR study ${ }^{2}$, the population studied was a prospective cohort of new patients, whose diagnosis was reevaluated after 6 months; the clinicians, who were from 21 centers in 10 countries, had considerable experience in the disease and met several times to agree on diagnosis standardization; the control group were patients with conditions mimicking PMR, including RA and noninflammatory shoulder conditions. The single-center study by Macchioni, et $a l^{6}$ was retrospective, patients were consecutively enrolled and followed by expert clinicians, diagnosis was confirmed at 12 months, and controls included mainly early RA and various other rheumatic conditions. Ozen's study ${ }^{4}$ was

See Validation of PMR classification criteria, page 893

Personal non-commercial use only. The Journal of Rheumatology Copyright @ 2016 . All rights reserved. 
Table 1. Performance of different sets of diagnostic/classification criteria. Values are expressed as percentages.

\begin{tabular}{|c|c|c|c|c|c|c|c|}
\hline & Ozen, et $a l^{4}$ & Weigand, et $a l^{5}$ & Macchioni, et $a l^{6}$ & Dasgupta, et $a l^{2}$ & Bird, $e t a l^{7}$ & Nobunaga, et $a l^{8}$ & Bird, et $a l^{9}$ \\
\hline \multicolumn{8}{|l|}{ Sensitivity (\%) } \\
\hline ACR/EULAR & 89.5 & 85.2 & 92.6 & 68 & & & \\
\hline Nobunaga & 73.7 & & 58.2 & & 67.8 & 93.1 & \\
\hline Jones and Hazleman & 47.4 & 83.3 & 63.1 & & 84.9 & 48 & \\
\hline Healey & 48.1 & 66.7 & 80.3 & & & 42.9 & \\
\hline \multicolumn{8}{|l|}{ Specificity (\%) } \\
\hline ACR/EULAR & 57.7 & & 81.5 & 78 & & & \\
\hline ACR/EULAR US & 52 & & 91.3 & 81 & & & \\
\hline Nobunaga & 83.1 & & 97.8 & & & 98.3 & \\
\hline Jones and Hazleman & 93.7 & & 96.7 & & & 100 & \\
\hline Healey & 89.4 & & 81.5 & & & 100 & \\
\hline
\end{tabular}

ACR/EULAR: American College of Rheumatology/European League Against Rheumatism; US: ultrasonography.

prospective and multicenter (18 centers), enrolled patients showed the same features as the ACR/EULAR study, and diagnosis was validated after 12 months. The rate of misclassification was similar; in the original ACR/EULAR study, $8 \%$ of patients moved from the PMR to the control cohort and $4.7 \%$ of controls were reclassified as PMR by the end of followup; and in Ozen's study $8.3 \%$ of patients with PMR changed diagnosis, with more than half reclassified as RA. As a result, it is difficult to find rough methodological differences between these studies that could explain the different results obtained. The only variables remaining are the percentage mix of controls and the clinician's ability to make a diagnosis and perform ultrasonography (US).

\section{Which Additional Aids Could Be Useful to Identify and Support the Correct Diagnosis?}

Ultrasonography. Is US the magic solution for this problem? In the original study ${ }^{2}$, the addition of US increased specificity from $78 \%$ to $81 \%$, but US decreased sensitivity from $68 \%$ to $66 \%$. In another study ${ }^{6}$, US highly increased specificity from $81.5 \%$ to $91.3 \%$, and did not change sensitivity. Conversely, Weigand, et $a l^{5}$ observed a decrease in sensitivity from $85.2 \%$ to $81.5 \%$. Ozen, et $a l^{4}$ found contrasting results, with an increase in sensitivity from $89.5 \%$ to $91.3 \%$ and a decrease in specificity from $57.7 \%$ to $52 \%$. And in the same report, US contributed best when used to differentiate PMR from noninflammatory shoulder disorders. US may increase our ability to classify PMR, but is probably not necessary for diagnosis because its effect on sensitivity is minimal or nonexistent. Other advanced imaging techniques, such as contrast-enhanced magnetic resonance imaging and positron emission tomography/computerized tomography, possibly share the same problem ${ }^{12}$.

Inflammatory markers. The ACR/EULAR criteria require the presence of abnormal ESR and/or CRP, considered as a dichotomous variable. In their cohort, Ozen, et al found that in establishing an ESR cutoff 2 times higher than the upper normal value, the best performance of sensitivity and specificity was achieved. When a CRP increase of at least 2 times was also set, specificity improved, but sensitivity slightly decreased. The reason why the authors of the ACR/EULAR criteria did not include CRP concentrations or ESR values in their set of criteria was that blood tests were not centralized or standardized. This was also the case for Ozen, et al's work and therefore their results, although theoretically sound, should be considered with caution. In the majority of elderly patients, however, mild degrees of systemic inflammation, often due to atherosclerosis, should be taken into account ${ }^{13}$. This situation could also justify why, in the cohort of Ozen, et al, most patients with noninflammatory conditions of the shoulders had elevated ESR (79\%) or CRP (57\%). What is more, several reports have shown that up to $10 \%$ to $20 \%$ of PMR patients present with normal inflammatory indices ${ }^{14}$. As a result, if only those patients with an increase of ESR or CRP are included in the PMR category, the correct diagnosis could be missed in about 1 in 5 . What is needed is another prospective study where laboratory examinations are centralized.

Response to glucocorticoids. For many years a good response to glucocorticoid (GC) therapy has been used as a confirmatory criterion, an approach that could be misleading, because other diseases, whether rheumatic or not, are improved by GC. In addition, it is not clear how to define this good response. The ACR/EULAR study reported it as over $75 \%$ global response to 15 to $20 \mathrm{mg} /$ day of prednisone. This result was not reached in $29 \%$ of patients at 4 weeks, which is contrary to the common opinion that patients with PMR always respond dramatically to GC. There was no association

$$
\text { Personal non-commercial use only. The Journal of Rheumatology Copyright } \subset \text { } 2016 \text {. All rights reserved. }
$$


between the scores obtained on either algorithm and the response to GC at 4 and 26 weeks, which suggests that GC response cannot be used as part of PMR classification.

Workup of patients. Another potential pitfall is the subjectivity of each physician in deciding on the diagnostic investigations for patients with suspected PMR. Standardization of diagnostic procedures was another aim of the ACR/EULAR criteria group, which involved a wide range of participants, including patients and physicians (rheumatologists, internists, and general practitioners) to emphasize different viewpoints. The 2015 recommendations for management of PMR ${ }^{15}$ provide a list of mandatory laboratory tests, which should be performed at baseline for all patients. These include CRP and/or ESR, rheumatoid factor and/or anticyclic citrullinated peptide antibodies, blood cell count, glucose, creatinine concentration, liver function tests, bone profile, and dipstick urinalysis. In addition, in selected instances, additional tests such as protein electrophoresis, thyroid stimulating hormone, creatine kinase, Vitamin D, antinuclear antibodies, anti-neutrophil cytoplasmic antibodies, or tuberculin skin tests should be performed. Adoption of these procedures should diminish diagnostic variability among clinicians.

The ACR/EULAR criteria seem to constitute progress in the classification of PMR, although more data are needed on their prospective evaluation in different settings, with an eye to standardizing the numerous variables at work.

\section{DARIO CAMELLINO, MD; MARCO A. CIMMINO, MD,}

Research Laboratory and Academic Division of Clinical Rheumatology, Department of Internal Medicine,

University of Genoa,

Genoa, Italy.

Address correspondence to Dr. D. Camellino, Research Laboratory and Academic Division of Clinical Rheumatology, Department of Internal Medicine, University of Genoa, Viale Benedetto XV, 6, 16132 Genoa, Italy.E-mail: dario.camel@gmail.com

\section{REFERENCES}

1. Caporali R, Montecucco C, Epis O, Bobbio-Pallavicini F, Maio T, Cimmino MA. Presenting features of polymyalgia rheumatica and rheumatoid arthritis with PMR-like onset: a prospective study. Ann Rheum Dis 2001;60:1021-4.
2. Dasgupta B, Cimmino MA, Maradit-Kremers H, Schmidt WA, Schirmer M, Salvarani C, et al. 2012 provisional classification criteria for polymyalgia rheumatica: a European League Against Rheumatism/ American College of Rheumatology collaborative initiative. Ann Rheum Dis 2012;71:484-92.

3. Aggarwal R, Ringold S, Khanna D, Neogi T, Johnson SR, Miller A, et al. Distinctions between diagnostic and classification criteria. Arthritis Care Res 2015;67:891-7.

4. Ozen G, Inanc N, Unal AU, Bas S, Kimyon G, Kisacik B, et al. Assessment of the new 2012 EULAR/ACR clinical classification criteria for polymyalgia rheumatica: a prospective multicenter study. J Rheumatol 2016;43:893-900.

5. Weigand S, Ehrenstein B, Fleck M, Hartung W. Joint involvement in patients with early polymyalgia rheumatica using high-resolution ultrasound and its contribution to the EULAR/ACR 2012 classification criteria for polymyalgia rheumatica. J Rheumatol 2014;41:730-4.

6. Macchioni P, Boiardi L, Catanoso M, Pazzola G, Salvarani C. Performance of the new 2012 EULAR/ACR classification criteria for polymyalgia rheumatica: comparison with the previous criteria in a single-centre study. Ann Rheum Dis 2014;73:1190-3.

7. Bird HA, Leeb BF, Montecucco CM, Misiuniene N, Nesher G, Pai S. A comparison of the sensitivity of diagnostic criteria for polymyalgia rheumatica. Ann Rheum Dis 2005;64:626-9.

8. Nobunaga M, Yoshioka K, Yasuda M, Shingu M. Clinical studies of polymyalgia rheumatica. A proposal of diagnostic criteria. Jpn J Med 1989;28:452-6.

9. Bird HA, Esselinckx W, Dixon AS, Mowat AG, Wood PH. An evaluation of criteria for polymyalgia rheumatica. Ann Rheum Dis 1979;38:434-9.

10. Chuang TY, Hunder GG, Ilstrup DM, Kurland LT. Polymyalgia rheumatica: a 10-year epidemiologic and clinical study. Ann Intern Med 1982;97:672-80.

11. Jones JG, Hazleman BL. Prognosis and management of polymyalgia rheumatica. Ann Rheum Dis 1981;40:1-5.

12. Camellino D, Cimmino MA. Imaging of polymyalgia rheumatica: indications on its pathogenesis, diagnosis and prognosis. Rheumatology 2012;51:77-86.

13. Ferrucci L, Corsi A, Lauretani F, Bandinelli S, Bartali B, Taub D, et al. The origins of age-related proinflammatory state. Blood 2005;105:2294-9.

14. Helfgott SM, Kieval RI. Polymyalgia rheumatica in patients with a normal erythrocyte sedimentation rate. Arthritis Rheum 1996;39:304-7.

15. Dejaco C, Singh YP, Perel P, Hutchings A, Camellino D, Mackie S, et al. 2015 Recommendations for the management of polymyalgia rheumatica: a European League Against Rheumatism/American College of Rheumatology collaborative initiative. Ann Rheum Dis 2015;74:1799-807.

J Rheumatol 2016;43:836-8; doi:10.3899/jrheum.160232 